\title{
Bioinformatics analysis of the expression of inducible nitric oxide synthases (iNOS/NOS2) in human glioma and its correlation with patients' prognoses
}

https://doi.org/10.1515/pteridines-2020-0019 received April 6, 2020; accepted June 3, 2020.

\begin{abstract}
Objective To evaluate the expression of inducible nitric oxide synthases (iNOS/NOS2) in human glioma and its correlation with patients' prognoses.
\end{abstract}

Methods IiNOS/NOS2 expression in tumor and corresponding normal tissues of glioma patients was analyzed using the TCGA database and the online analysis tool GEPIA. The mutation statuses of iNOS/NOS2 genes were also explored in the TCGA database using cBioPortal. Co-expressed genes relevant to iNOS/NOS2 were screened by LinkedOmics. Gene ontology (GO) and KEGG pathway enrichment for iNOS/NOS2 and co-expressed genes was performed using LinkedOmics. Overall survival (OS) and disease-free survival (DFS) outcomes between iNOS/NOS2 mRNA high and low expression groups were compared using a log-rank test. Twenty-two glioma patients who underwent operation were included in the present work. A real-time PCR assay was used to detect iNOS/NOS2 mRNA expression in tumor tissue and normal brain tissue.

Results There was no statistical difference in iNOS/ NOS2 mRNA expression levelss between tumor and normal tissues of glioma. A real-time PCR assay indicated that iNOS/NOS2 mRNA expression in tumor tissue and normal brain tissues were not statistical difference $(p>0.05)$. A mutation rate of $0.8 \%$ for the iNOS/NOS2 gene was found using 1044 glioma patients from two datasets. The mutation types include deep deletion $(0.4 \%)$, truncating

\footnotetext{
*Corresponding author: Xueqing Zhang, Department of Neuroelectrophysiology, Tianjin Huanhu Hospital, Tianjin 300350 PR China, E-mail: zlphhyydsl@163.com

Liping Zhang, Mei Feng, Department of Neuroelectrophysiology, Tianjin Huanhu Hospital, Tianjin 300350 PR China

Huanyu Wang, Department of Neurosurgery, Tianjin Huanhu Hospital, Tianjin 300350 PR China

\# Liping Zhang \& Huanyu Wang contribute equally to this work
}

$(0.2 \%)$ and missense $(0.2 \%)$. The top positive and negative co-expressed gene with iNOS/NOS2 were COL25A1 $\left(r_{\text {pearson }}=0.4734, p<0.05\right)$ and ALCAM $\left(r_{\text {pearson }}=0.4734\right.$, $\mathrm{p}<0.05)$, respectively. For KEGG pathway analysis, iNOS/ NOS2 was mainly enriched in calcium signaling pathway, Wnt signaling pathway, GnRH signaling pathway, HIF-1 signaling pathway and pathways in cancer. The overall survival $(\mathrm{HR}=2.0, \mathrm{p}<0.05)$ and disease-free survival ( $\mathrm{HR}=1.6, \mathrm{p}<0.05)$ values were significantly different between iNOS/NOS2 high and low expression groups.

Conclusion OS and DFS were significantly decreased in high iNOS/NOS2 mRNA expression groups. iNOS/NOS2 can be used as a poor prognostic biomarker for glioma.

Keywords: Bioinformatics analysis; inducible nitric oxide synthases; glioma; prognosis.

\section{Introduction}

Nitric oxide (NO) is a kind of bioactive molecule in the human body. Recent studies have shown that NO is closely related to tumorigenesis and development of a variety of malignant tumors [1-3]. NO in tumor tissues is mainly synthesized by inducible nitric oxide synthase (iNOS). iNOS, known as inducible type NOS, is noncalcium-dependent $[4,5]$. It exists in the cytoplasm of leukocytes, glial cells and many other cells. When the cells are stimulated, such as by inflammation, degeneration, and tumorigenesis, iNOS is activated and expressed. The NO produced by iNOS widely participates in various pathological processes [6].

Most studies have shown that expression of iNOS in gliomas is significantly higher than that of normal tissues, and iNOS expression is positively correlated with the degree of malignancy. However, Giannopoulou et al. [7] found that expression of iNOS in astrocytomas increased 
with increased pathology grading of I, II and III. However, iNOS expression levels were significantly decreased in grade IV astrocytomas. Therefore, iNOS expression patterns in human glioma and their correlation with patients' prognoses were not yet completely clear.

\section{Methods}

\section{Bioinformatics analysis}

Nitric oxide synthases (iNOS/NOS2) expression of tumor and corresponding normal tissues of glioma patients were analyzed using the TCGA databases and the online analysis tool GEPIA [8]. The mutation statuses of iNOS/ NOS2 genes were also explored in the TCGA database using cBioPortal. Co-expressed genes relevant to iNOS/ NOS2 were screened by LinkedOmics [9]. Gene ontology (GO) and KEGG pathway enrichment for iNOS/NOS2 and co-expressed genes was performed using LinkedOmics in the aspects of biological function(BP), cellular component(CC) and molecular function(MF).

\section{Real-time PCR assay}

Twenty-two glioma patients who underwent operation were included in the present work. This work was approved by the ethical committee of Tianjin Huanhu Hospital. A real-time PCR assay was used to detect iNOS/ NOS2 mRNA expression in tumor tissue and normal brain tissue. Total RNA from tissues and cells was isolated using RNA Tri zol (Invitrogen, Carlsbad, CA, USA). Using agarose gel electrophoresis and BioPhotometer Plus (Eppendorf AG, Hamburg, Germany), RNA integrity and amount were assayed. Then, $2 \mu \mathrm{g}$ of total RNA was reverse transcribed into cDNA using reverse transcriptase (Invitrogen) according to the manufacturer's protocols. The primers of iNOS/NOS2 were Forward primers: 5'-'GGATGACTTTCGAGGACATGC-3'" and Reverse primers: 5'-GGGCCCTCTGGTCATACTTTT-3'. PCR was performed using a ABI 7500 Real-Time PCR system (Applied Biosystems, Austin, TX, USA). Relative expressions of mRNAs were calculated using 2- $\Delta \Delta \mathrm{Ct}$ method.

\section{Survival analysis}

According to the median iNOS/NOS2 expression level in tumor tissue of the glioma cases, patients were divided into high ( $>=$ median expression) and low $(<$ median expression) iNOS/NOS2 expression groups. The OS and DFS between the high and low iNOS/NOS2 expression groups were compared by log-rank test and Cox regression analysis and shown with a Kaplan-Meier survival plot.

\section{Statistical analysis}

Stata15.0 software (https://www.stata.com/) was used for data analysis. Measurement data was expressed as $\bar{x} \pm s$, and a t test was used to make comparisons between groups. Counting data was expressed by number, and a chi-square test was used to make comparisons between groups. iNOS/NOS2 expression, mutation and survival analysis was performed using the corresponding online software of service. Two tailed $\mathrm{P}<0.05$ was considered statistically significant.

\section{Results}

\section{iNOS/NOS2 mRNA relative expression}

iNOS/NOS2 mRNA levels in glioma are shown in Figure 1. There was no statistical difference in iNOS/NOS2 mRNA expression level between tumor and normal tissues of glioma. iNOS/NOS2 mRNA expression levels in other carcinomas are shown in Figure 2. iNOS/NOS2 mRNA seem to be upregulated in colorectal cancer and prostate carcinoma.

\section{Real-time PCR assay}

The real-time PCR assay indicated that iNOS/NOS2 mRNA expression in tumor tissues of glioma patients was downregulated compared to expression in normal brain tissue, but without statistical difference ( $p>0.05)$, Figure 3 .

\section{iNOS/NOS2 mutation analysis}

iNOS/NOS2 gene mutation analysis was performed using the TCGA databases and the online analysis tool cBioPortal. A total mutation rate of $0.8 \%$ for iNOS/NOS2 genes was found using 1044 glioma patients from two datasets (Figure 3A). The mutation types include deep deletion $(0.4 \%)$, truncating $(0.2 \%)$ and missense $(0.2 \%)$. 


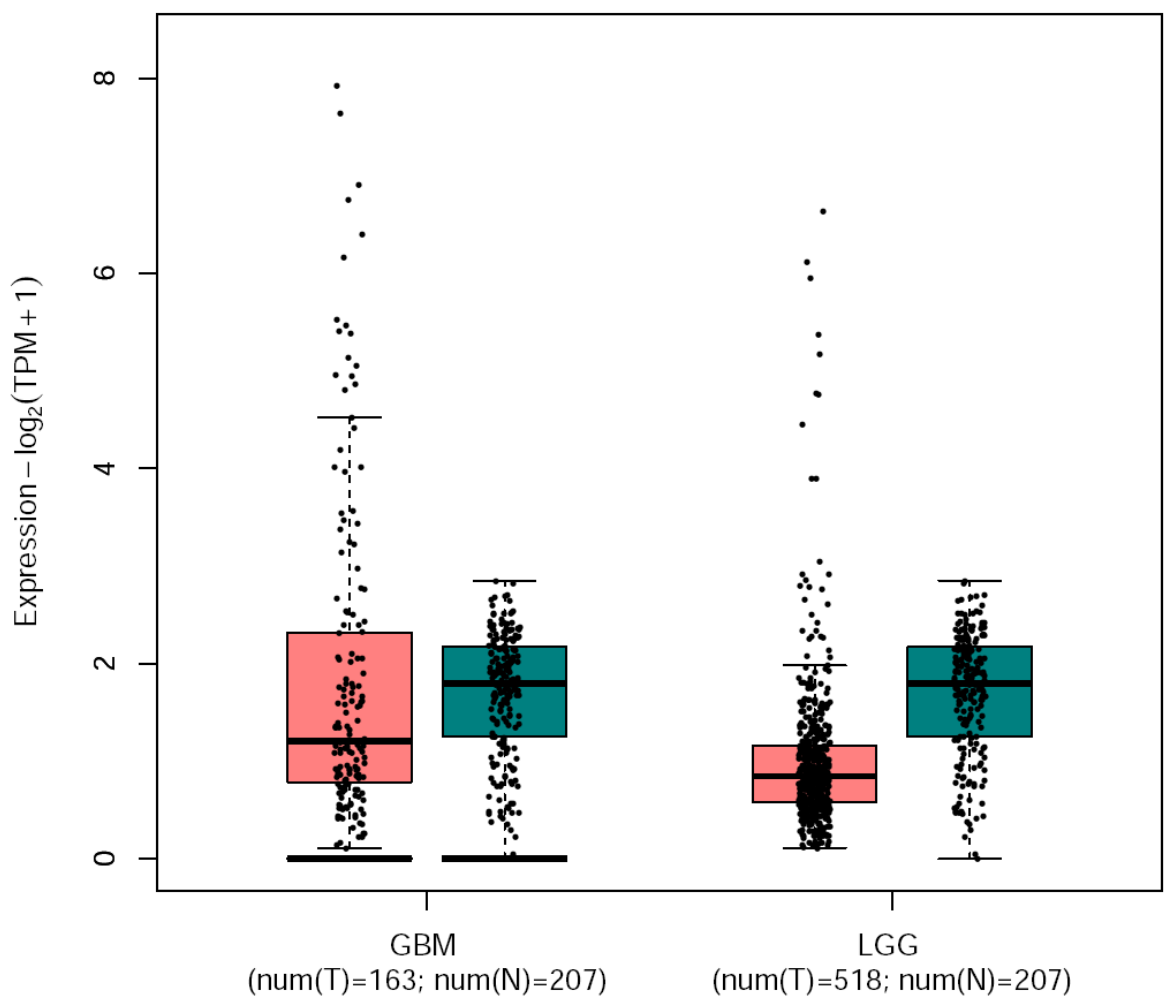

Figure 1: iNOS/NOS2 mRNA relative expression scatter plot of tumor tissues and corresponding normal tissues of glioma patients.

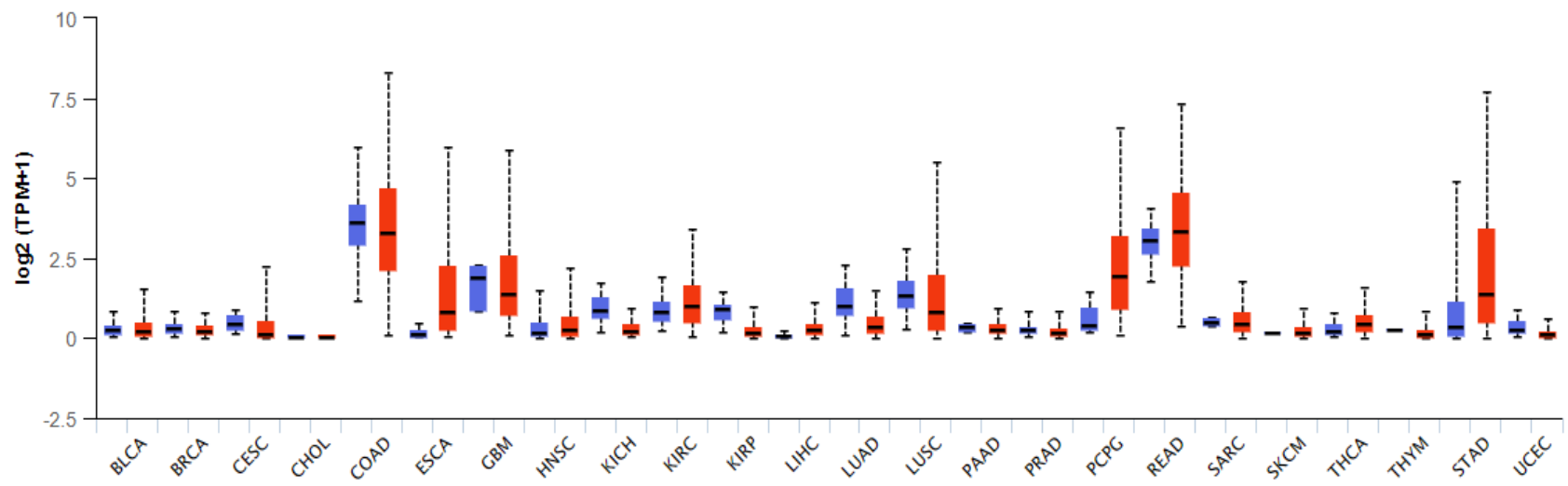

Figure 2: Figure 1. iNOS/NOS2 mRNA relative expression levels in multiple carcinomas.

\section{Co-expression analysis}

The top 50 positive and negative co-expressed genes for iNOS/NOS2 are shown in Figure 5. The top positive and negative co-expressed genes for iNOS/NOS2 were COL25A1 $\left(\mathrm{r}_{\text {pearson }}=0.4734, \mathrm{p}<0.05\right)$ and ALCAM $\left(\mathrm{r}_{\text {pearson }}=0.4734\right.$, $\mathrm{p}<0.05)$, respectively, Figure 5.

\section{GO enrichment analysis}

Enrichment results for biological process (BP), cellular component (CC) and molecular function (MF) analyses of iNOS/NOS2 and co-expressed genes are shown in Tables 1-3. With regard to KEGG pathways, iNOS/NOS2 was mainly enriched in calcium signaling pathway, Wnt signaling pathway, GnRH signaling pathway, HIF-1 signaling pathway and pathways in cancer, Table 4. 


\section{iNOS/NOS2 expression and prognosis}

According to the median iNOS/NOS2 expression in tumor tissues of glioma subjects, patients were divided into iNOS/NOS2 high and low expression groups. The overall survival $(\mathrm{HR}=2.0, \mathrm{p}<0.05)$ and disease-free survival $(\mathrm{HR}=1.6, \mathrm{p}<0.05)$ were significantly different between the iNOS/NOS2 high and low expression groups. The overall survival and disease-free survival in the iNOS/NOS2 high

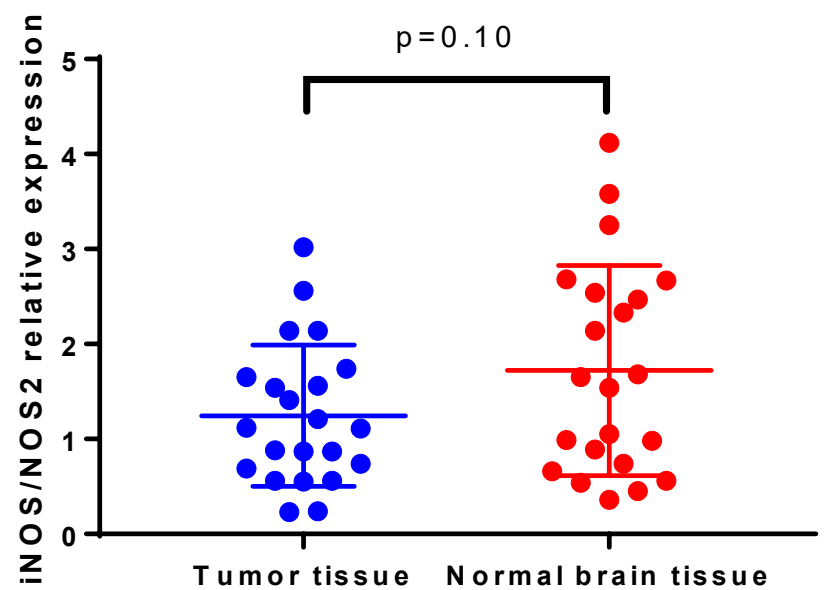

Figure 3: Scatter plot of iNOS/NOS2 mRNA expression levels in tumor tissues and normal brain tissues of glioma patients. expression group were significantly lower than those of the low expression group, Figure 6.

\section{Discussion}

Epidemiological data indicates that gliomas are the most frequently clinically diagnosed primary tumor of the central nervous system [10]. Gliomas are generally divided into 4 types (I to IV) according to the pathology characteristics and biological behavior of the tumor cells. The general prognosis is poor, especially for grade IV gliomas, with a median survival period of 1 to 1.5 years after diagnosis, even if comprehensive treatment is provided [11-13].

As in other solid malignant tumors, the inflammatory environment plays an important role in the tumorigenesis of gliomas [14, 15]. Nitric oxide (NO) is a radical molecule that acts as a ubiquitous messenger and can be induced by an inducible isoform (iNOS or NOS2) and sustained at high levels in tumor cells. Several studies [16-18] have found that LPS, IFN - $y$, TNF - $\alpha$ and other cytokines can upregulate expression of iNOS. Mattace et al. [19] found that PDTC and TLCK, two inhibitors of NF- $\mathrm{kB}$, can significantly reduce the ability of LPS, IFN $-y$, TNF $-\alpha$ to promote expression of iNOS. Therefore, it has been indicated that LPS, IFN $-\gamma$, TNF $-\alpha$ regulate expression of iNOS through the NF-kB pathway. Kim et al. [20] found

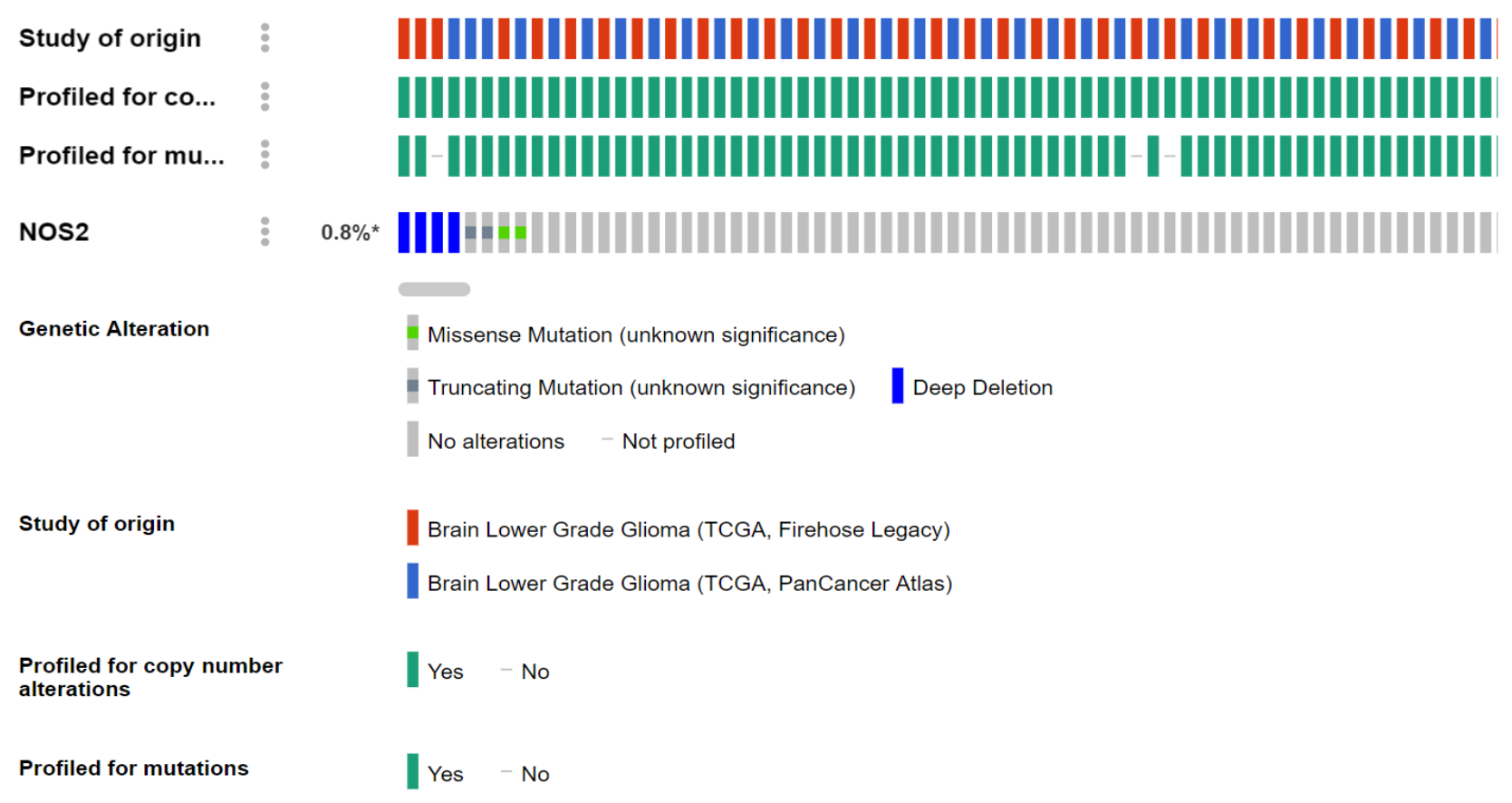

Figure 4: iNOS/NOS2 mutation analysis using TCGA databases and the online analysis tool cBioPortal. 


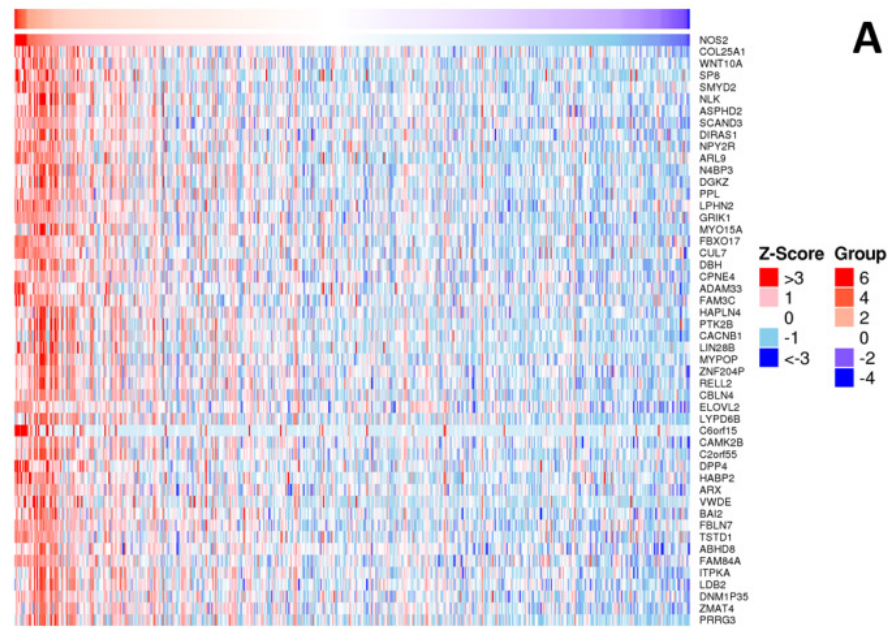

A

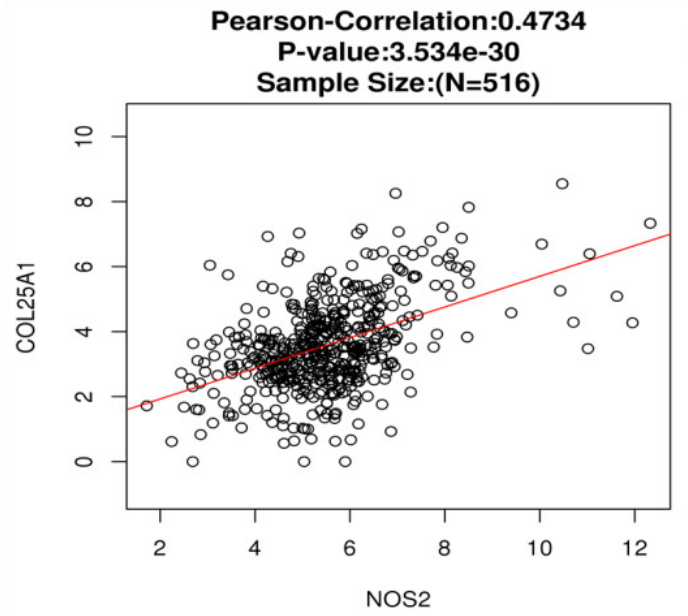

B
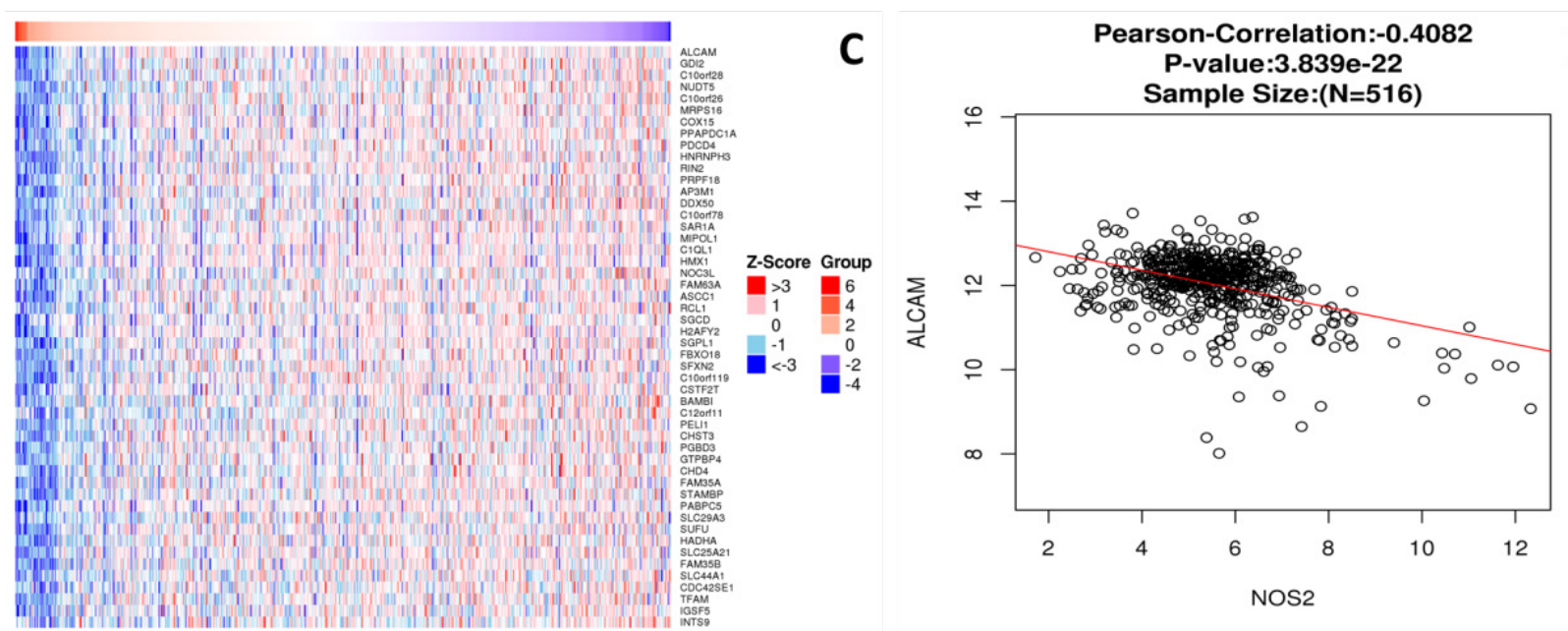

Figure 5: Co-expressed genes relevant to iNOS/NOS2 analysis (A: Heat-map of genes positively correlated with iNOS/NOS2; B: Top gene positively correlated with iNOS/NOS2; C: Heat-map of genes negatively correlated with iNOS/NOS2; D: Top gene negatively correlated with iNOS/NOS2).
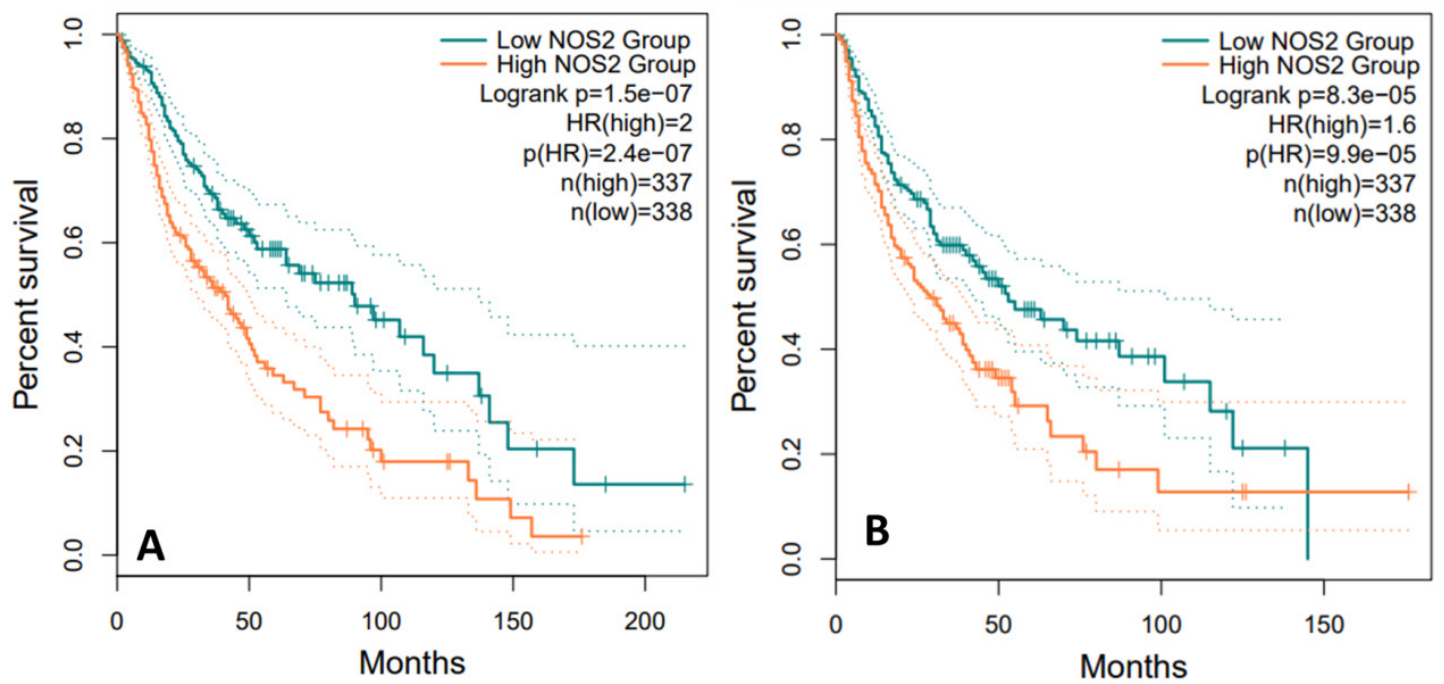

Figure 6: Survival curve of iNOS/NOS2 expression and prognosis in patients with glioma (A: Overall survival of iNOS/NOS2 low and high expression groups; B: Disease-free survival of iNOS/NOS2 low and high expression groups) 
Table 1: Biological process enrichment for iNOS/NOS2 and co-expressed genes.

\begin{tabular}{llllc}
\hline Description & Size & Expect & Ratio & P-value \\
\hline peptidyl-aspartic acid hydroxylation & 4 & 0.0032 & 312.56 & 0.0032 \\
positive regulation of circadian sleep/wake cycle, non-REM sleep & 5 & 0.004 & 250.05 & 0.00399 \\
peptidyl-aspartic acid modification & 5 & 0.004 & 250.05 & 0.00399 \\
axonogenesis involved in innervation & 6 & 0.0048 & 208.38 & 0.00479 \\
positive regulation of circadian sleep/wake cycle, sleep & 6 & 0.0048 & 208.38 & 0.00479 \\
positive regulation of dopamine secretion & 6 & 0.0048 & 208.38 & 0.00479 \\
peptidyl-lysine monomethylation & 7 & 0.0056 & 178.61 & 0.00559 \\
sebaceous gland development & 7 & 0.0056 & 178.61 & 0.00559 \\
negative regulation of feeding behavior & 9 & 0.0072 & 138.92 & 0.00718 \\
negative regulation of synaptic transmission, glutamatergic & 9 & 0.0072 & 138.92 & 0.00718 \\
\hline
\end{tabular}

Table 2: Cellular component enrichment for iNOS/NOS2 and co-expressed genes.

\begin{tabular}{lllll}
\hline Description & Size & Expect & Ratio & P-value \\
\hline intercellular canaliculus & 4 & 0.00792 & 126.25 & 0.0079 \\
3M complex & 5 & 0.0099 & 101 & 0.00986 \\
kainate selective glutamate receptor complex & 5 & 0.0099 & 101 & 0.00986 \\
Cul7-RING ubiquitin ligase complex & 5 & 0.0099 & 101 & 0.00986 \\
chromaffin granule membrane & 6 & 0.01188 & 84.167 & 0.01182 \\
invadopodium membrane & 6 & 0.01188 & 84.167 & 0.01182 \\
chromaffin granule & 8 & 0.01584 & 63.125 & 0.01574 \\
NMDA selective glutamate receptor complex & 11 & 0.02178 & 45.909 & 0.02158 \\
platelet dense granule lumen & 13 & 0.02574 & 38.846 & 0.02545 \\
apical dendrite & 14 & 0.02772 & 36.071 & 0.02738 \\
\hline
\end{tabular}

Table 3: Molecular function enrichment for iNOS/NOS2 and co-expressed genes.

\begin{tabular}{lllll}
\hline Description & Size & Expect & Ratio & P-value \\
\hline dopamine beta-monooxygenase activity & 2 & 0.00532 & 188.04 & 0.00531 \\
Rab GDP-dissociation inhibitor activity & 2 & 0.00532 & 188.04 & 0.00531 \\
8-oxo-dGDP phosphatase activity & 2 & 0.00532 & 188.04 & 0.00531 \\
ADP-sugar diphosphatase activity & 2 & 0.00532 & 188.04 & 0.00531 \\
nitric-oxide synthase activity & 3 & 0.00798 & 125.36 & 0.00796 \\
peptide YY receptor activity & 3 & 0.00798 & 125.36 & 0.00796 \\
peptide-aspartate beta-dioxygenase activity & 3 & 0.00798 & 125.36 & 0.00796 \\
oxidoreductase activity & 4 & 0.01064 & 94.019 & 0.0106 \\
tetrahydrobiopterin binding & 4 & 0.01064 & 94.019 & 0.0106 \\
ADP-ribose diphosphatase activity & 4 & 0.01064 & 94.019 & 0.0106 \\
\hline
\end{tabular}


Table 4: KEGG pathway enrichment for iNOS/NOS2 and co-expressed genes.

\begin{tabular}{lllll}
\hline Description & Size & Expect & Ratio & P-value \\
\hline Calcium signaling pathway & 181 & 0.42576 & 9.395 & 0.00069 \\
Wnt signaling pathway & 143 & 0.33637 & 8.9187 & 0.00417 \\
GnRH signaling pathway & 92 & 0.21641 & 9.2418 & 0.0192 \\
HIF-1 signaling pathway & 97 & 0.22817 & 8.7655 & 0.02121 \\
Phosphatidylinositol signaling system & 97 & 0.22817 & 8.7655 & 0.02121 \\
Melanogenesis & 101 & 0.23758 & 8.4183 & 0.02288 \\
Phospholipase D signaling pathway & 140 & 0.32931 & 6.0732 & 0.0418 \\
Adrenergic signaling in cardiomyocytes & 144 & 0.33872 & 5.9045 & 0.04399 \\
Arginine biosynthesis & 20 & 0.04705 & 21.256 & 0.04607 \\
Oxytocin signaling pathway & 149 & 0.35049 & 5.7064 & 0.04679 \\
Cushing syndrome & 152 & 0.35754 & 5.5938 & 0.0485 \\
Fatty acid elongation & 23 & 0.0541 & 18.484 & 0.05281 \\
Biosynthesis of unsaturated fatty acids & 23 & 0.0541 & 18.484 & 0.05281 \\
Tuberculosis & 176 & 0.414 & 4.831 & 0.063 \\
Proteoglycans in cancer & 196 & 0.46104 & 4.338 & 0.07609 \\
Tyrosine metabolism & 36 & 0.08468 & 11.809 & 0.08149 \\
Arginine and proline metabolism & 44 & 0.1035 & 9.6619 & 0.09873 \\
Fatty acid metabolism & 44 & 0.1035 & 9.6619 & 0.09873 \\
Pathways in cancer & 519 & 1.2208 & 2.4574 & 0.11775 \\
Glycerolipid metabolism & 53 & 0.12467 & 8.0212 & 0.11776 \\
\hline & & & & \\
\hline
\end{tabular}

that IL-1 $\beta$ is an early protein that promotes expression of iNOS in rat C6 glioma. It can regulate expression of iNOS through the p38 MAPK and NF-kB pathways. Xu et al. [21] also confirmed that $\mathrm{p} 38$ MAPK, rather than $\mathrm{p} 42$ / p44mapk, is involved in the expression of iNOS in C6 glioma.

In the present work, we investigated the expression of inducible nitric oxide synthases (iNOS/NOS2) in human glioma and its correlation with patients' prognoses through bioinformatics analysis. We found that iNOS/ NOS2 mRNA expression levels were not different between tumor and normal tissues of glioma patients. However, the tumor tissues had a trend of upregulated expression compared to normal tissues. Further analysis of iNOS/ NOS2 in other carcinomas showed that the difference was not statistically significant for most solid cancers. Therefore, we suspect that the biological function of iNOS/ NOS2 in carcinoma maybe not depend on concentration level, a hypothesis which is partly supported by previous studies. We also noticed a total mutation ratte of $0.8 \%$ for iNOS/NOS2 based on analysis of the TCGA datasets. The mutation types include deep deletion (0.4\%), truncating
$(0.2 \%)$ and missense $(0.2 \%)$. This indicated that iNOS/ NOS2 mutations are not common in glioma. Prognosis analysis demonstrated that the overall survival $(\mathrm{HR}=2.0$, $\mathrm{p}<0.05)$ and disease-free survival $(H R=1.6, p<0.05)$ values were significantly different between the iNOS/NOS2 high and low expression groups. The OS and DFS was significantly decreased in the high iNOS/NOS2 mRNA expression group. iNOS/NOS2 can be used as a poor prognostic biomarker for glioma.

The potential molecular mechanism by which iNOS/ NOS2 affects glioma patients' prognoses may include promotion of neovascularization and inhibition of tumor cell apoptosis. NO plays contradictory roles in tumor progression, and its function in promoting or inhibiting cancer depends on the tumor type. NO levels in normal tissues are relatively stable and play a certain physiological role. In malignant tumor tissues, iNOS is activated by various cytokines, thus releasing more NO. The role of $\mathrm{NO}$ in tumorigenesis is complex. It has been reported that the production of a certain amount of NO in tumors can increase tumor blood flow and promote 
angiogenesis, thus promoting tumor growth [22]. However, high concentrations of NO have toxic effects on tumor cells, promote cell apoptosis, block tumor angiogenesis and play an anti-tumor role. Generally, NO in tumor tissue mainly plays a role in promoting tumor growth. The molecular mechanism of interaction between NO and VEGF may be as follows: (1) NO, as an important signal transduction factor in the binding process of VEGF and its receptor produced by tumor cells, plays an important role in inducing tumor angiogenesis [23]; (2) NO promotes VEGF-induced increase of vascular permeability, increases the nutritional supply of tumor tissue, and ultimately promotes tumor growth [24].

iNOS is also closely related to apoptosis of tumor cells. In mammalian cells, NO is involved in apoptosis as an intracellular biological messenger or cytotoxic effector [25]. NO initiates apoptosis or cell survival signals according to its concentration and intracellular redox state. Most scholars [26-28] believe that NO produced by malignant tumor cells not only plays a role in increasing tumor blood supply and promoting tumor angiogenesis, but also inhibits tumor cell apoptosis.

\section{In conclusion}

INOS is widely involved in the angiogenesis, proliferation, apoptosis and chemotherapy tolerance of glioma, which are closely related to the carcinogenesis and development of glioma. Therefore, iNOS may be a new potential target for comprehensive treatment of glioma. However, most of these results were based on data mining from several databases, and further cell and clinical experiments are needed to validate the findings.

Data Availability Statement: The datasets generated during or analysed during the current study are available from the corresponding author on reasonable request.

\section{Conflict of interest: Authors state no conflict of interest}

\section{References}

1. Counter C. Evaluating The Role Of Nitric Oxide Synthase In Oncogenic Ras-Driven Tumorigenesis. Redox Biol. 2015 Aug;5:417.

2. Hussain SP, He P, Subleski J, Hofseth LJ, Trivers GE, Mechanic $L$, et al. Nitric oxide is a key component in inflammationaccelerated tumorigenesis. Cancer Res. 2008 Sep;68(17):71306.
3. Inano H, Onoda M. Nitric oxide produced by inducible nitric oxide synthase is associated with mammary tumorigenesis in irradiated rats. Nitric Oxide. 2005 Feb;12(1):15-20.

4. Cinelli MA, Do HT, Miley GP, Silverman RB. Inducible nitric oxide synthase: Regulation, structure, and inhibition. Med Res Rev. 2020 Jan;40(1):158-89.

5. Robinson MA, Baumgardner JE, Otto CM. Oxygen-dependent regulation of nitric oxide production by inducible nitric oxide synthase. Free Radic Biol Med. 2011 Dec;51(11):1952-65.

6. Lind M, Hayes A, Caprnda M, Petrovic D, Rodrigo L, Kruzliak $P$, et al. Inducible nitric oxide synthase: good or bad? Biomed Pharmacother. 2017 Sep;93:370-5.

7. Giannopoulou E, Ravazoula P, Kalofonos H, Makatsoris T, Kardamakis D. Expression of HIF-1alpha and iNOS in astrocytic gliomas: a clinicopathological study. In Vivo. 2006 MayJun;20(3):421-5.

8. Tang Z, Li C, Kang B, Gao G, Li C, Zhang Z. GEPIA: a web server for cancer and normal gene expression profiling and interactive analyses. Nucleic Acids Res. 2017 Jul;45 W1:W98-102.

9. Vasaikar SV, Straub P, Wang J, Zhang B. LinkedOmics: analyzing multi-omics data within and across 32 cancer types. Nucleic Acids Res. 2018 Jan;46 D1:D956-63.

10. Siegel RL, Miller KD, Jemal A. Cancer statistics, 2018. CA Cancer J Clin. 2018 Jan;68(1):7-30.

11. Ghotme KA, Barreto GE, Echeverria V, Gonzalez J, Bustos RH, Sanchez M, et al. Gliomas: New Perspectives in Diagnosis, Treatment and Prognosis. Curr Top Med Chem. 2017;17(12):1438-47.

12. Alireza M, Amelot A, Chauvet D, Terrier LM, Lot G, Bekaert O. Poor Prognosis and Challenging Treatment of Optic Nerve Malignant Gliomas: Literature Review and Case Report Series. World Neurosurg. 2017 Jan;97:751.e1-6.

13. Gupta T, Sarin R. Poor-prognosis high-grade gliomas: evolving an evidence-based standard of care. Lancet Oncol. 2002 Sep;3(9):557-64.

14. Liu X, Yang J, Deng W. The inflammatory cytokine IL-22 promotes murine gliomas via proliferation. Exp Ther Med. 2017 Mar;13(3):1087-92.

15. Deininger MH, Seid K, Engel S, Meyermann R, Schluesener HJ. Allograft inflammatory factor-1 defines a distinct subset of infiltrating macrophages/microglial cells in rat and human gliomas. Acta Neuropathol. 2000 Dec;100(6):673-80.

16. Yang SH, Li L, Xie YQ, Yao Y, Gao CY, Liao LH, et al. IFN$Y$-STAT1-iNOS Induces Myeloid Progenitors to Acquire Immunosuppressive Activity. Front Immunol. 2017 Sep;8:1192.

17. Singh D, Kumar V, Singh C. IFN-y regulates xanthine oxidasemediated iNOS-independent oxidative stress in maneb- and paraquat-treated rat polymorphonuclear leukocytes. Mol Cell Biochem. 2017 Mar;427(1-2):133-43.

18. Salim T, Sershen CL, May EE. Investigating the Role of TNF- $\alpha$ and IFN- $\gamma$ Activation on the Dynamics of iNOS Gene Expression in LPS Stimulated Macrophages. PLoS One. 2016 Jun;11(6):e0153289.

19. Mattace Raso G, Esposito E, Iacono A, Pacilio M, Coppola A, Bianco $G$, et al. Leptin induces nitric oxide synthase type II in C6 glioma cells. Role for nuclear factor-kappaB in hormone effect. Neurosci Lett. 2006 Mar;396(2):121-6.

20. Kim YJ, Hwang SY, Oh ES, Oh S, Han IO. IL-1beta, an immediate early protein secreted by activated microglia, induces iNOS/ 
NO in C6 astrocytoma cells through p38 MAPK and NF-kappaB pathways. J Neurosci Res. 2006 Oct;84(5):1037-46.

21. Xu X, Malave A. P38 MAPK, but not p42/p44 MAPK mediated inducible nitric oxide synthase expression in C6 glioma cells. Life Sci. 2000 Nov;67(26):3221-30.

22. Niu XJ, Wang ZR, Wu SL, Geng ZM, Zhang YF, Qing XL. Relationship between inducible nitric oxide synthase expression and angiogenesis in primary gallbladder carcinoma tissue. World J Gastroenterol. 2004 Mar;10(5):725-8.

23. Ziche M, Morbidelli L, Choudhuri R, Zhang HT, Donnini S, Granger HJ, et al. Nitric oxide synthase lies downstream from vascular endothelial growth factor-induced but not basic fibroblast growth factor-induced angiogenesis. J Clin Invest. 1997 Jun;99(11):2625-34.

24. Song ZJ, Gong P, Wu YE. Relationship between the expression of iNOS,VEGF,tumor angiogenesis and gastric cancer. World J Gastroenterol. 2002 Aug;8(4):591-5.

25. Li Y, Gu J, Liu Y, Long H, Wang G, Yin G, et al. iNOS participates in apoptosis of spinal cord neurons via $p-B A D$ dephosphorylation following ischemia/reperfusion (I/R) injury in rat spinal cord. Neurosci Lett. 2013 Jun;545:117-22.

26. Deepa M, Sureshkumar T, Satheeshkumar PK, Priya S. Antioxidant rich Morus alba leaf extract induces apoptosis in human colon and breast cancer cells by the downregulation of nitric oxide produced by inducible nitric oxide synthase. Nutr Cancer. 2013;65(2):305-10.

27. Kong G, Kim E, Kim W, Lee Y, Lee J, Paik S, et al. Inducible Nitric Oxide Synthase (iNOS) Immunoreactivity and Its Relationship to Cell Proliferation, Apoptosis, Angiogenesis, Clinicopathologic Characteristics, and Patient Survival in Pancreatic Cancer. Int J Gastrointest Cancer. 2001;29(3):13340.

28. Chung P, Cook T, Liu K, Vodovotz Y, Zamora R, Finkelstein S, et al. Overexpression of the human inducible nitric oxide synthase gene enhances radiation-induced apoptosis in colorectal cancer cells via a caspase-dependent mechanism. Nitric Oxide. 2003 Mar;8(2):119-26. 